\title{
Prevalência de Inatividade Física e Fatores Associados em Estudantes do Estado de Pernambuco
}

\section{Prevalence of Physical Inactivity and Associated Factors in Students from the State of Pernambuco}

\author{
Lailah Maria Luiza Gonzaga Cavalcanti ${ }^{1}$ \\ Alison Oliveira da Silva ${ }^{2}$ \\ Fernanda Cunha Soares ${ }^{3}$ \\ Mauro Gomes Virgílio Barros ${ }^{2,4}$ \\ Jorge Bezerra ${ }^{2,4}$
}

\section{RESUMO}

Objetivo: Estimar a prevalência de adolescentes inativos fisicamente e identificar fatores associados a esse comportamento. Metodologia: Estudo transversal de abrangência estadual, realizado com 6002 adolescentes do ensino médio do Estado Pernambuco. Foi utilizado o questionário "Global school-based student health survey" (GSHS) aplicado em forma de entrevista coletiva. Os possíveis fatores associados pesquisados foram o sexo, a idade, a cor da pele, o local de residência, a escolaridade materna, o trabalho, o turno, a série e a participação nas aulas de educação física. O desfecho utilizado foi inatividade física, categorizada em sim/não. Foi realizada a análise descritiva, o teste Qui-quadrado e para análise multivariada foi utilizada a regressão logística binária. Resultado: A prevalência de adolescentes classificados como inativos fisicamente foi de $56,0 \%$, sendo a proporção maior de meninas, classificadas como fisicamente inativas $66,6 \%$ quando comparado aos meninos (38,6\%). As meninas tiveram 3 (IC:2,6$3,2)$ vezes mais chance de serem inativas quando comparadas aos meninos. Os adolescentes que não participavam das aulas de educação física tiveram 2,4 (IC:2,1-2,8) vezes mais chance de serem inativos. Conclusão: A prevalência de adolescentes inativos fisicamente foi alta, particularmente entre as meninas. Ademais, não participar das aulas de educação física esteve associado a maiores prevalências de inatividade física, o que ressalta a importância dessas aulas para os adolescentes. Assim, são necessárias estratégias em saúde pública visando difundir e estimular a adesão à prática de atividades físicas pelos adolescentes

\section{DESCRITORES}

Exercício. Adolescente. Educação.

\begin{abstract}
Objetive: To estimate the prevalence of physically inactive adolescents and identify factors associated with this behavior. Methodology: Cross-sectional study with 6002 high school adolescents in the state of Pernambuco. The "Global School-based Student Health Survey" (GSHS) questionnaire was applied in the form of collective interview. The possible associated factors surveyed were gender, age, skin color, place of residence, maternal education, work, and participation in physical education classes. The outcome was physical inactivity categorized as yes/no. Descriptive analysis, Chi-square test and binary logistic regression were used for multivariate analysis. Result: The prevalence of adolescents classified as physically inactive was $56.0 \%$, with a higher proportion of girls classified as physically inactive $66.6 \%$ when compared to boys (38.6\%). Girls were 3 (Cl: 2.6-3.2) times more likely to be inactive when compared to boys and adolescents who did not participate in physical education classes were $2.4(\mathrm{Cl}: 2.1-2.8)$ times more likely to be inactive. Conclusion: The prevalence of physically inactive adolescents was high, particularly among girls. In addition, not participating in physical education classes was associated with higher prevalence of physical inactivity, which highlights the importance of these classes for adolescents. Thus, public health strategies are needed to disseminate and encourage adherence to physical activity by adolescents.
\end{abstract}

\section{DESCRIPTORS}

Exercise. Adolescent. Education.

${ }^{1}$ Graduada em Educação Física, estagiária do Grupo de Pesquisa em Estilo de Vida e Saúde (GPES), Escola Superior de Educação Física, Universidade de Pernambuco, Recife, Pernambuco, Brasil.

${ }^{2}$ Mestre em Educação Física - Doutorando do Programa de Pós-Graduação em Neurociência, Faculdade de Medicina, Universidade Federal de Pernambuco, Recife, Pernambuco, Brasil.

${ }^{3}$ Doutora em Odontopediatria, Pós-doutoranda em Hebiatria do Grupo de Pesquisa em Estilo de Vida e Saúde (GPES), Escola Superior de Educação Física, Universidade de Pernambuco, Recife, Pernambuco, Brasil.

${ }^{4}$ Doutor em Ciências do Movimento Humano, Escola Superior de Educação Física, Universidade de Pernambuco, Recife, Pernambuco, Brasil.

${ }^{5}$ Doutor em Educação Física, Escola Superior de Educação Física, Universidade de Pernambuco, Recife, Pernambuco, Brasil. 
A prática de atividade física e os seus benefícios tem sido documentada como meio para obtenção de uma melhor saúde, prevenção de doenças, especialmente as do sistema cardiovascular e como intermediadora para manutenção ou aderência a um estilo de vida mais favorável à saúde ${ }^{1,2}$. Por essa razão, em 2004, o Center for Disease Control and Prevention emitiu uma recomendação de que os programas de intervenção nessa área devem ser iniciados na infância e na adolescência.

A adolescência é um período de importância para classificar comportamentos de saúde, pois as principais causas de doença e de morte neste período de desenvolvimento podem ser prevenidas. Globalmente, $81 \%$ dos adolescentes com idade entre 11-17 anos foram insuficientemente ativos fisicamente em $2010^{3}$. Estudos têm demonstrado um declínio em cerca de 7\% por ano nos níveis de atividade física durante a adolescência ${ }^{4}$. No Brasil, de acordo com uma revisão sistemática, a exposição a baixos níveis de atividade física pode variar de $39 \%$ a $93 \%$ entre os adolescentes ${ }^{2}$.

De acordo com a World Health Organization $(\mathrm{WHO})^{3}$, a inatividade física é um dos principais fatores de risco de morte, de $20 \%$ a $30 \%$ em comparação com pessoas suficientemente ativas, juntando-se ao grupo das doenças crônicas não transmissíveis (DCNs). Diversos fatores podem causar baixo nível de atividade física, como a ociosidade durante o tempo de lazer, o comportamento sedentário na escola e em casa e o aumento no uso de transporte passivos ${ }^{3}$.
No Brasil, alguns estudos identificaram diferenças regionais quanto às taxas de prevalência de inatividade física ${ }^{2,5}$, revelando divergências quanto aos níveis de atividade em diferentes localidades. Portanto, devido a carência de estudos que avaliam este contexto no Nordeste do Brasil ${ }^{6}$, o objetivo do presente estudo foi estimar a prevalência de adolescentes inativos fisicamente e identificar os fatores associados a esse comportamento.

\section{METODOLOGIA}

Trata-se de um estudo transversal, de base escolar e abrangência estadual, realizado em 2016, que faz parte de um projeto intitulado "Prática de Atividades Físicas e Comportamentos de Risco à Saúde em Estudantes do Ensino Médio no Estado de Pernambuco: Estudo de tendência temporal (2006 - 2011 - 2016) - Projeto Atitude, realizado pelo Grupo de Pesquisa em Estilo de Vida e Saúde (GPES), vinculado à Escola Superior de Educação Física da Universidade de Pernambuco (ESEF-UPE). O projeto foi aprovado pelo Comitê de Ética de Pesquisa em Seres Humanos da Universidade de Pernambuco (CAAE:56341416.9.0000.5192 / CEP-UPE). Todos os pais ou responsáveis assinaram o Termo de consentimento livre esclarecido. Serão descritas a seguir apenas as informações relevantes para este estudo, a metodologia estendida foi descrita por Carvalho et al. ${ }^{17}$.

A população alvo deste estudo envolveu estudantes com idade entre 14 e 19 anos de ambos os sexos, regularmente matriculados em escolas da Rede Pública Estadual de 
ensino médio do Estado de Pernambuco. Para o cálculo amostral foi considerado um intervalo de confiança de $95 \%$, um erro máximo tolerável de $2 \%$ e uma prevalência estimada das variáveis de $50 \%$, além de um deff de 2 , considerando que o processo amostral foi por conglomerado em 2 estágios. A amostra final foi inflada em $20 \%$, considerando as perdas e recusas.

Os dados foram coletados por meio do questionário adaptado e validado Global School-based Student Health Survey (GSHS) proposto pela $\mathrm{WHO}^{7}$, com o objetivo de avaliar comportamentos de risco em adolescentes. Foram utilizadas neste estudo as variáveis: sexo (masculino e feminino); idade; cor da pele (branco, não branco); local de residência (urbano, rural); escolaridade materna ( $\leq 8$ anos, >8 anos); trabalho (sim, não); turno (diurno, noturno, integral); série ( $1^{\mathrm{a}}$ série, $2^{\mathrm{a}}$ série, $3^{a}$ série) e participação nas aulas de educação física (sim, não).

A recomendação de atividade física para os adolescentes, segundo a $\mathrm{WHO}^{3}$ propõe a prática de atividades físicas de intensidade vigorosa e/ou moderada, dentro ou fora da escola, de forma estruturada ou não-estruturada por ao menos, 300 minutos/semana. Portanto, foi classificado como fisicamente inativo o adolescente que relatou fazer menos de 300 minutos/semana de atividade física.

A análise de dados foi realizada por meio do programa SPSS para Windows (versão 20). A análise feita foi realizada utilizando a distribuição de frequência (absoluta e relativa). A regressão logística binária bruta foi empregada para analisar quais fatores foram associados com a inatividade física e, em seguida, todas as variáveis com um valor de $p<0,05$ foram testadas em um modelo ajustado (regressão ajustada). Ao final, considerou-se significativamente associado aos desfechos, sob análise, aqueles cujo o valor de $p$ foi inferior a 0,05 .

\section{RESULTADOS}

Foram entrevistados 6314 adolescentes, dos quais 312 foram excluídos pois não estavam na faixa etária entre 14 a 19 anos. Dos 6002 adolescentes, 30\% tinham 16 anos, $91,5 \%$ eram solteiros e $53,4 \%$ se declaravam pardos. $38,6 \%$ cursavam o $1^{\circ}$ ano, $46,0 \%$ estudavam em turno integral, $87,0 \%$ não trabalhavam e $80,0 \%$ residiam na zona urbana (Tabela 1).

A prevalência de adolescentes classificados como fisicamente inativos foi de $56,0 \%$, sendo a proporção maior de meninas $(66,6 \%)$ quando comparadas aos meninos (38,6\%). Os resultados da análise multivariada demonstraram que, dos fatores que foram significativos na análise bruta, como a idade, a escolaridade materna e a série, perderam a significância após a análise ajustada. No modelo ajustado, as meninas tiveram 2,8 mais chances (IC:2,63,2 ) de serem inativas quando comparadas aos meninos. O trabalho mostrou-se um fator de proteção para inatividade física $(O R=0,8$; IC: 0,7-0,9). Adolescentes que não participavam das aulas de educação física tiveram 2,4 mais chances (IC:2,1-2,8) de serem inativos quando comparados aos que participavam. 
Tabela 1. Características sociodemográficas e escolares dos adolescentes estudantes da rede pública de ensino de Pernambuco, estratificado por sexo

\begin{tabular}{|c|c|c|c|c|c|c|}
\hline \multirow{2}{*}{ Variável } & \multicolumn{2}{|c|}{ Meninos } & \multicolumn{2}{|c|}{ Meninas } & \multicolumn{2}{|c|}{ Todos } \\
\hline & $\mathrm{n}$ & $\%$ & $n$ & $\%$ & $\mathrm{n}$ & $\%$ \\
\hline \multicolumn{7}{|l|}{ Idade (anos) } \\
\hline 14 & 67 & 2,5 & 115 & 3,5 & 183 & 3,1 \\
\hline 15 & 456 & 17,1 & 696 & 21,1 & 1155 & 19,2 \\
\hline 16 & 775 & 29,0 & 1015 & 30,8 & 1794 & 29,9 \\
\hline 17 & 804 & 30,1 & 916 & 27,8 & 1728 & 28,8 \\
\hline 18 & 433 & 16,2 & 396 & 12,0 & 842 & 14,0 \\
\hline 19 & 137 & 5,1 & 160 & 4,8 & 300 & 5,0 \\
\hline \multicolumn{7}{|l|}{ Estado Civil } \\
\hline Solteiro (a) & 2.472 & 92,8 & 2978 & 90,4 & 5478 & 91,4 \\
\hline Casado (a) & 54 & 2,0 & 153 & 4,6 & 209 & 3,5 \\
\hline Outro & 137 & 5,1 & 165 & 5,0 & 304 & 5,1 \\
\hline \multicolumn{7}{|l|}{ Cor da Pele } \\
\hline Branco (a) & 567 & 21,3 & 697 & 21,1 & 1270 & 21,2 \\
\hline Preto (a) & 439 & 16,5 & 369 & 11,2 & 816 & 13,6 \\
\hline Pardo (a) & 1378 & 51,7 & 1813 & 54,9 & 3207 & 53,4 \\
\hline Amarelo (a) & 156 & 5,9 & 292 & 8,8 & 450 & 7,5 \\
\hline Indígena (a) & 127 & 4,8 & 132 & 4,0 & 260 & $\overline{4,3}$ \\
\hline \multicolumn{7}{|l|}{ Turno } \\
\hline Diurno & 1054 & 39,3 & 1299 & 39,2 & 2368 & 39,2 \\
\hline Noturno & 411 & 15,3 & 459 & 13,9 & 881 & 14,6 \\
\hline Integral & 1215 & 45,4 & 1554 & 46,9 & 2777 & 46,1 \\
\hline \multicolumn{7}{|l|}{ Série } \\
\hline $1^{\circ}$ & 1039 & 38,8 & 1271 & 38,4 & 2.323 & 38,6 \\
\hline $2^{\circ}$ & 951 & 35,5 & 1148 & 34,6 & 2.112 & 35,1 \\
\hline $3^{\circ}$ & 690 & 25,7 & 893 & 27,0 & 1.591 & 26,4 \\
\hline \multicolumn{7}{|l|}{ Trabalho } \\
\hline Sim & 473 & 17,8 & 296 & 9,0 & 781 & 13,0 \\
\hline Não & 2.185 & 82,2 & 3004 & 91,0 & 5.210 & 87,0 \\
\hline \multicolumn{7}{|c|}{ Escolaridade Materna } \\
\hline$\leq 8$ anos & 950 & 42,3 & 1363 & 48,4 & 2.330 & 46,7 \\
\hline$>8$ anos & 1.294 & 57,7 & 1454 & 51,6 & 2.763 & 54,3 \\
\hline \multicolumn{7}{|c|}{ Local de Residência } \\
\hline Urbano & 2.143 & 80,6 & 2622 & 79,6 & 4.788 & 80,0 \\
\hline Rural & 515 & 19,4 & 673 & 20,4 & 1.194 & 20,0 \\
\hline
\end{tabular}


Tabela 2. Fatores associados à inatividade física em adolescentes estudantes da rede pública de ensino de Pernambuco.

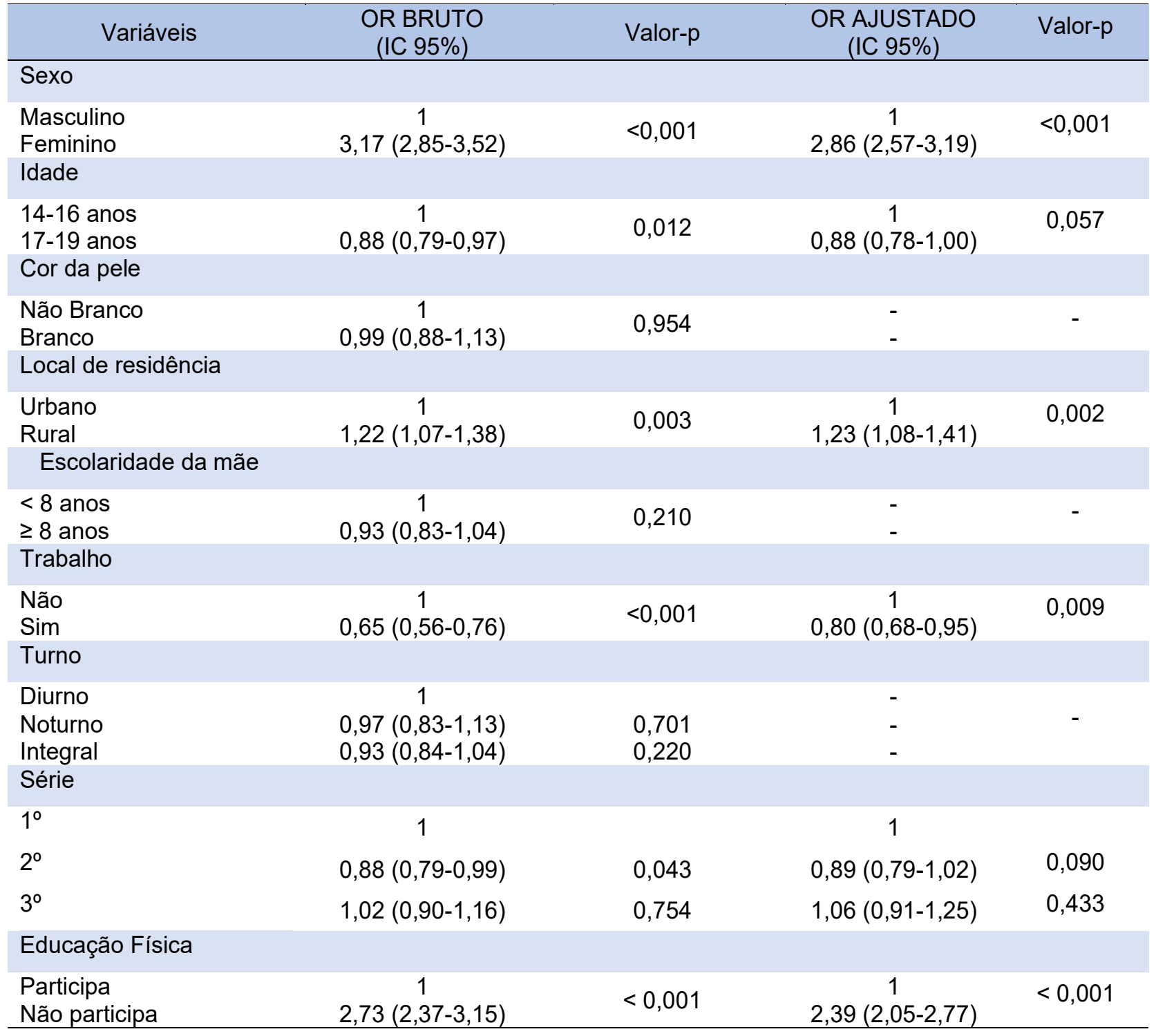




\section{DISCUSSÃO}

Este estudo transversal analisou a prevalência de inatividade física e seus possíveis fatores associados em uma amostra de adolescentes pernambucanos. Foi observado que 6 , a cada 10 adolescentes, foram classificados como inativos fisicamente, sendo esta estimativa de prevalência um dado importante para a compreensão de um dos comportamentos relacionados à saúde nessa população. Uma maior proporção de meninas inativas foi observada, quando comparadas aos meninos. Adolescentes que não participavam das aulas de educação física também se mostraram mais fisicamente inativos.

Resultados similares foram observados em um estudo com dados da Pesquisa Nacional de Saúde do Escolar (PeNSE), com 60.973 adolescentes do $9^{\circ}$ ano, das 26 capitais brasileiras e do Distrito Federal, onde a proporção de jovens suficientemente ativos foi de $43,1 \%{ }^{9}$. As divergências quanto as prevalências entre as regiões do Brasil, pode ser intermediada pelos fatores ambientais, ligados à urbanização como a poluição, a falta de parques, de calçadas e de instalações desportivas/recreativas, o medo da violência e do crime em áreas ao ar livre ${ }^{3}$, e até mesmo ao clima, que no Nordeste, apresenta predominantemente, temperaturas elevadas.

A diferença na prevalência de inatividade física entre os sexos apresentada neste estudo também foi relatada por outros autores. Hallal et al. ${ }^{9}$ observaram uma maior prevalência de meninos fisicamente ativos $(56,2 \%)$ em comparação com as meninas $(31,3 \%)$.
Em uma meta-análise sobre a prevalência de inatividade física entre adolescentes brasileiros, os autores verificaram que as taxas de prevalência variaram de $2 \%$ a $80 \%$ para o sexo masculino e de $14 \%$ para $91 \%$ para o feminino e, ainda, as menores taxas de prevalência foram encontradas na Região Sul e, as maiores, nas Regiões Norte e Nordeste ${ }^{5}$. Essa diferença entre o sexo e os níveis de prática de atividade física tem sido explicadas pelas diferenças culturais, comportamentais, psicológicas e de maturação envolvidas nas fases da adolescência ${ }^{10,11}$.

No presente estudo, não participar das aulas de educação física foi um fator associado a maiores níveis de inatividade física nos adolescentes. Acredita-se que a participação na educação física contribua para o aumento da atividade física habitual, como evidenciado em outros estudos ${ }^{12-16}$. É importante salientar que a educação física escolar contribui com uma pequena parcela para alcançar a atividade física desejada para os adolescentes, assim, devendo ser estimulada a prática, também, fora do ambiente escolar.

A interpretação destes achados deve ser realizada com cautela, em face de algumas limitações. O delineamento adotado não permite concluir a causalidade, ademais, as informações foram fornecidas pelos próprios estudantes e, portanto, existe a possibilidade de viés de registro e de memória. Como ponto forte do estudo, destaca-se o fato de ter sido conduzido com uma amostra precisa e representativa dos adolescentes estudantes do ensino médio do Estado de Pernambuco. Ademais, os dados foram coletados mediante 
a utilização de um questionário que foi previamente testado e apresentou um nível de reprodutibilidade de moderado a alto.

\section{CONCLUSÃO}

A prevalência de adolescentes inativos fisicamente foi alta, particularmente entre as meninas. Ademais, não participar das aulas de educação física esteve associado a maiores prevalências de inatividade física, o que ressalta a importância dessas aulas para os adolescentes. Diante destas constatações, são necessárias estratégias em saúde pública visando difundir e estimular a adesão à prática de atividades físicas, visto que esta se relaciona à adoção de hábitos de vida saudáveis, auxiliando na promoção da saúde, na diminuição de riscos para o desenvolvimento de doenças e, assim, uma maior expectativa de vida nesta população.

\section{REFERÊNCIAS}

1. Janssen I, LeblancAG. Systematic review of the health benefits of physical activity and fitness in school-aged children and youth. Int J Behav Nutr Phys Act. 2010; 7:2-16.

2. Tassitano RM, Bezerra J, Cecília M, Tenório M, Virgílio M, Barros G De, et al. Atividade Física Em Adolescentes Brasileiros: Uma Revisão Sistemática. Rev Bras Cineantropometria Desempenho Hum. 2007; 9(1):55-60.

3. World Health Organization. Physical activity [Internet]. 2018.

4. Dumith SC, Gigante DP, Domingues MR, lii HWK. Physical activity change during adolescence : a systematic review and a pooled analysis. Int J Epidemiol. 2011; 40:685-98.

5. Barufaldi LA, Abreu G de A, Coutinho ESF, Bloch KV. Meta-analysis of the prevalence of physical inactivity among Brazilian adolescents. Cad Saude Publica [Internet]. 28(6):1019-32.

6. Barbosa Filho VC, Campos W, Lopes A da S. Epidemiology of physical inactivity, sedentary behaviors, and unhealthy eating habits among Brazilian adolescents: a systematic review. Cien Saude Colet. 2014;19(1):173-94.

7. World Health Organization. Global school-based student health survey (GSHS) [Internet]. 2005.
8. World Health Organization. Global Recommendations on Physical Activity for Health. 2010. 1-58 p.

9. Hallal PC, Knuth AG, Cruz DKA, Mendes MI, Malta DC Prática de atividade física em adolescentes brasileiros. Cienc e Saude Coletiva. 2010;15(2):3035-3042.

10. Cheah YK, Lim HK, Kee CC, Ghazali SM. Factors associated with participation in physical activity among adolescents in Malaysia. Int J Adolesc Med Health. 2015; 2015(4):419-427.

11. Thompson AM, Baxter-jones ADG, Mirwald RL, Bailey DA. Comparison of Physical Activity in Male and Female Children : Does Maturation Matter ? Med Sci Sport Exerc. 2003; 35(10):1684-90.

12. Jerônimo R, Augusto D, Silva S, Oliveira AC. Low Physical Activity Levels and Associated Factors in Brazilian Adolescents From Public High Schools. J Phys Act Heal. 2014;11:1438-1445.

13. Atividade física e comportamento sedentário em adolescentes estudantes do ensino médio. Rev Bras Epidemiol. 2010;13(1):105-17.

14. Farias Júnior JC de, Lopes A da S, Mota J, Hallal PC. Prática de atividade física e fatores associados em adolescentes no Nordeste do Brasil. Rev Saude Publica. 2012;46(3):505-15.

15. Bergmann GG, Bergman MLDA, Marques AC, Hallal PC. Prevalence of physical inactivity and associated factors among adolescents from public schools in Uruguaiana , Rio Grande do Sul State, Brazil. Cad Saúde Pública. 2013; 29(11):2217-29. 
16. Coledam DHC, Ferraiol PF, Pires Jr R, Ribeiro EAG, Ferreira MAC, de Oliveira AR. Agreement between two cutoff points for physical activity and associated factors in young individuals. Rev Paul Pediatr. 2014; 32(3):215-22.

17. Carvalho PD, Barros MVG, Lima RA, Santos CM, Mélo EN. Health risk behaviors and psychosocial distress indicators in high school students. Cad. Saúde Pública. 2011; 27(11):2095-2105.

\section{CORRESPONDÊNCIA}

Lailah Maria Luiza Gonzaga Cavalcant

Rua Arnóbio Marquês, 310 - Santo Amaro,

Recife - PE, CEP: 50100-130.

E-mail: lailahcavalcanti@gmail.com 